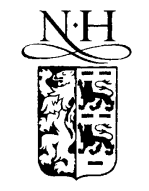

\title{
Oxygen sensitivity of erbium-doped AlN films probed by site selective spectroscopy
}

\author{
J.C. Oliveira ${ }^{\mathrm{a}, *}$, A. Cavaleiro ${ }^{\mathrm{a}}$, M.T. Vieira ${ }^{\mathrm{a}}$, L. Bigot ${ }^{\mathrm{b}}$, C. Garapon ${ }^{\mathrm{b}}$, \\ B. Jacquier ${ }^{b}$, J. Mugnier ${ }^{b}$ \\ a ICEMS, Grupo de Materiais e Engenharia de Superficies, Departamento de Engenharia Mecanica, Universidade de Coimbra, \\ Pinhal de Marrocos, 3030 Coimbra, Portugal \\ ${ }^{\mathrm{b}}$ Laboratoire de Physico-Chimie des Matériaux Luminescents, UMR-CNRS 5620, Université Lyon1, 10 rue André-Marie Ampère, \\ Campus de la Doua, 69622 Villeurbanne Cedex, France
}

Received 8 December 2002; accepted 24 February 2003

\begin{abstract}
In the aim of better understand the influence of oxygen on the luminescent properties of Er:AlN films, two samples synthesized by radiofrequency reactive magnetron sputtering, have been analysed by site-selective spectroscopy around $1.55 \mu \mathrm{m}$ and compared to a reference aluminosilicate sample. The erbium content in the films was estimated to 3.3 at. $\%$ and oxygen content ranges from 6.2 at.\% to 58.6 at.\% (determination by EPMA). Line narrowing study was performed for values 6.2 at. $\%$ and 22.5 at.\%. Both films were annealed at $1075 \mathrm{~K}$ for $1 \mathrm{~h}$. Site-selective spectroscopy was carried out using a Ti:sapphire laser light with typical linewidth of $2 \mathrm{GHz}$ tuned around $980 \mathrm{~nm}$ as the excitation source. The samples were cooled down to $1.5 \mathrm{~K}$ in a liquid-helium bath cryostat and their ${ }^{4} \mathrm{I}_{13 / 2} \leftrightarrow{ }^{4} \mathrm{I}_{15 / 2}$ luminescence was detected using a high-sensitivity germanium-cooled detector. It appears that the site distribution of the Er:AlN samples is not continuous as in classical aluminosilicate glass but presents a rupture that suggests the existence of two kinds of sites for erbium: oxygen site and nitrogen site. These observations can easily be interpreted within the framework of the nephelauxetic effect that explains a shift of the site distribution to the longer wavelengths with an increase of boundings bonding covalency.
\end{abstract}

(C) 2003 Elsevier B.V. All rights reserved.

Keywords: Er:AlN; Photoluminescence; FLN

\section{Introduction}

There is, in the scientific community, a permanent interest for rare-earth-doped semiconductors

\footnotetext{
* Corresponding author. Address: Department of Physics, University of Lisbon, Campo Grande, Ed. C1, Lisboa 1700, Portugal.

E-mail address: joao.oliveira@dem.uc.pt (J.C. Oliveira).
}

due to the possibility they offer to provide thin-film electro-luminescent devices in the visible and also in the optical telecommunication range. As an example, erbium-doped semiconductors have been grown and good capabilities have been reported for $\mathrm{GaN}$ or nanocrystalline silicon [1,2]. In the case of erbium, it has been shown that wide band gap materials are required to improve luminescence intensity at room temperature [3]. Therefore, $\mathrm{AlN}$ (gap equal to $6.2 \mathrm{eV}$ ) is a good candidate for 
erbium doping and luminescent properties of erbium-doped AlN at room temperature have already been reported [4,5]. Moreover, erbiumdoped AlN films have been proposed as optical sensors to evaluate wear and corrosion in coated mechanical components [6]. However, it has been shown that luminescence intensity and spectral profile is very dependent of synthesis conditions and post-deposition treatments.

In the aim of better understand the influence of synthesis process on luminescent properties, this paper investigates more accurately the influence of oxygen on the luminescent properties of Er:AlN films by site-selective spectroscopy around $1.55 \mu \mathrm{m}$ [7,8]. Two samples synthesized by magnetron RF sputtering under different oxygen pressures have been analysed and compared to a reference aluminosilicate sample. It appears a strong sensitivity of the site distribution of erbium dopants depending on the oxygen content. More precisely, it is shown that the site distribution is not continuous as in the case of the aluminosilicate sample and can be related to the existence of oxygen and nitrogen environments for erbium sites.

\section{Experimental}

The Er:AlN samples studied in this work were films deposited on $\mathrm{Si}$ substrates using radiofrequency reactive magnetron sputtering from a $99.99 \%$ pure $\mathrm{Al}$ target. The deposition system has already been described elsewhere [6]. All depositions were achieved with a discharge power of 600 $\mathrm{W}$ ( $\varnothing$ of the target $=100 \mathrm{~mm}$ ), a total pressure of $0.74 \mathrm{~Pa}$ and a relative nitrogen pressure (PN2/ PTot) equal to 0.5 . The oxygen content in the films was varied by using a precision valve to introduce a controlled leak of air in the deposition chamber. In this way, thin films with an oxygen content ranging from 6.2 to 58.8 at.\%, as measured by electron probe microanalysis (EPMA), were deposited. The incorporation of erbium in the AlN films was achieved by partially covering the aluminium target with an adapted number of small metallic erbium pieces. The erbium concentration can be adjusted by modifying the Er/Al area ratio on the target or on the position of erbium pieces compared to the magnetron ring. The erbium concentration in the samples was estimated to 3.3 at. $\%$ by EPMA. Annealing of the films was carried out at $1075 \mathrm{~K}$ in a quartz tube, previously evacuated down to an ultimate vacuum pressure of less than $3 \times 10^{-3} \mathrm{~Pa}$, for $1 \mathrm{~h}$ in a dynamic hydrogenated argon atmosphere.

Infrared room temperature photoluminescence (PL) was excited by the $980 \mathrm{~nm}$ radiation of a CW Titanium-sapphire laser while the PL signals were analysed through a $1 / 4 \mathrm{~m}$ monochromator, detected using a liquid-nitrogen cooled $\mathrm{Ge}$ detector and measured by a standard lock-in amplifier. Two samples have been tested using high-resolution spectroscopy, namely site-selective spectroscopy. In these experiments, a sample is excited using the Ti:sapphire laser light with typical linewidth of $2 \mathrm{GHz}$. The wavelength used for the experiment is tuned around $980 \mathrm{~nm}$ in accordance with the ${ }^{4} \mathrm{I}_{15 / 2} \rightarrow{ }^{4} \mathrm{I}_{11 / 2}$ absorption transition. The samples were mounted in a liquid-helium bath cryostat that enables to reach temperature as low as $1.5 \mathrm{~K}$, the temperature used for all the measurements. Assuming a fast and efficient relaxation from the ${ }^{4} \mathbf{I}_{11 / 2}$ (the maximum phonon energy in AlN being close to $900 \mathrm{~cm}^{-1}$ as reported in [8]) the ${ }^{4} \mathbf{I}_{13 / 2} \rightarrow{ }^{4} \mathbf{I}_{15 / 2}$ luminescence emitted by the sample was collected and focused on the entrance slit of the $1 / 4 \mathrm{~m}$ monochromator. The signal was then processed as mentioned above. As a reference of aluminium oxide environment, an aluminosilicate glass fabricated by MCVD process has also been examined.

\section{Results and discussion}

The evolution of the erbium emission band at room temperature around $1.55 \mu \mathrm{m}$, as a function of oxygen content in the films, is shown in Fig. 1. As can be observed, the intensity as well as the profile of the emission at room temperature changes when the oxygen content increases. Moreover, a shift of the peak intensity to the longer wavelengths is observed as the oxygen level rises (see inset of Fig. 1).

Two Er:AlN films have been tested using siteselective spectroscopy. The oxygen content of the 


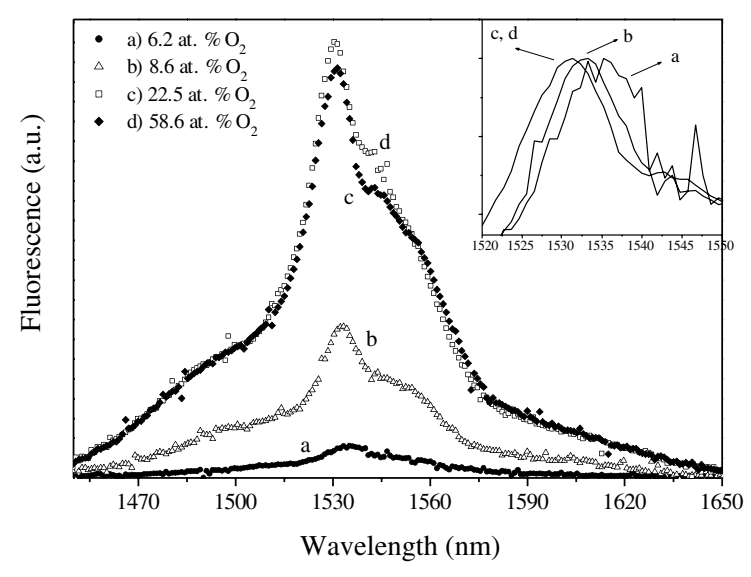

Fig. 1. Evolution of the erbium PL emission at room temperature around $1.55 \mu \mathrm{m}$ as a function of oxygen content in the films. In the inset, an enlargement of the normalized main peak is depicted.

oxygen rich film (which will be referred as the $\mathrm{O}^{+}$ film) was estimated to 22.5 at.\% while the oxygen poorer film (which will be referred as the $\mathrm{O}^{-}$film) presented an oxygen content of 6.2 at.\%.

The principle of the fluorescence line narrowing (FLN) experiment is the following: at very low temperature, it is possible to probe the luminescent properties of the erbium ions belonging to different sites using a spectrally narrow laser. Actually, in a crystal and moreover in a glass, the luminescent ions are located in different crystallographic sites characterised by different crystal field electronic environment. Each class of sites possess its own luminescent properties, namely: position of the energy levels, broadening, lifetime... At low temperature, the emission and absorption bands are narrow due to the small homogeneous broadening and the small number of energy levels populated. Using a spectrally narrow laser, it is then possible to selectively excite the ions: each excitation wavelength corresponding to a given class of sites. Because the nonresonant excitation occurs in the ${ }^{4} \mathrm{I}_{9 / 2}{ }^{4} \mathrm{I}_{11 / 2}$ excited state, rapid relaxation from this excited state to the emitting state $\left({ }^{4} \mathrm{I}_{11 / 2}{ }^{4} \mathrm{I}_{13 / 2}\right)$ contributes to a residual broadening. That is the reason why, in all the spectra, measured lines are still broad as compare to resonant fluorescence line narrowing (RFLN) experiment [9]. In the case of our samples, the evolutions of the $1.55 \mu \mathrm{m}$ emis- sion spectrum as a function of the excitation wavelength are reported in Figs. 2-4. For all the samples, at shorter excitation wavelength, a broadband emission is obtained, resulting from the nonselective excitation of all the erbium ions. Decreasing the excitation energy (increasing the wavelength), it is then possible to achieve a selective



Fig. 2. Evolution of the ${ }^{4} I_{13 / 2} \leftrightarrow{ }^{4} I_{15 / 2}$ emission spectrum at 1.5 $\mathrm{K}$ as a function of excitation wavelength for the aluminosilicate glass.

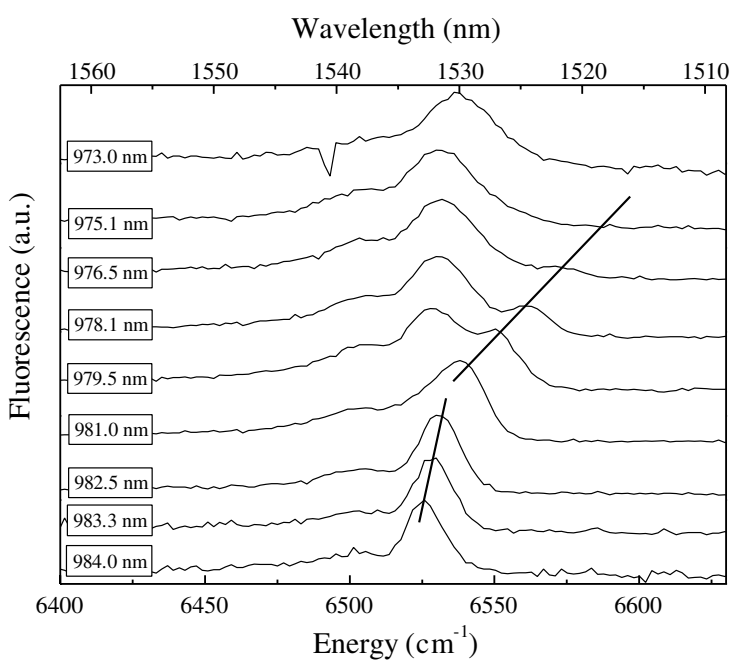

Fig. 3. Evolution of the ${ }^{4} \mathrm{I}_{13 / 2} \leftrightarrow{ }^{4} \mathrm{I}_{15 / 2}$ emission spectrum at 1.5 $\mathrm{K}$ as a function of excitation wavelength for the $\mathrm{O}^{+}$film. 




Fig. 4. Evolution of the ${ }^{4} I_{13 / 2} \leftrightarrow{ }^{4} I_{15 / 2}$ emission spectrum at 1.5 $\mathrm{K}$ as a function of excitation wavelength for $\mathrm{O}^{-}$film.

excitation characterised by a sharp emission band of which the position varies with the excitation wavelength: it is attributed to the zero-phonon transition of the probed class of sites (transition between the bottom Stark levels of the two energy manifold involved). At longer wavelength, no more ions are excited and no signal is detected. The spectral range over which the zero-phonon transition is recorded gives information about the site distribution of the erbium ions in the studied matrix: the larger this spectral range, the more inhomogeneous is the environment of the erbium ions.

As it appears in Fig. 2, the aluminosilicate glass exhibits a continuous site distribution extending from roughly 972 to $982 \mathrm{~nm}$ (around $100 \mathrm{~cm}^{-1}$ ). The energy of the maximum PL intensity of the selective excitation emission varies linearly with excitation energy (see Fig. 5). This is interpreted as an evidence for a unique kind of environment for erbium ions that is slightly modified from one ion to another. It can be related to the Gaussian profile of the site distribution already pointed out by RFLN [10].

The two Er:AlN samples present very different behaviours. The site distribution for $\mathrm{O}^{+}$is discontinuous, as can be observed in Fig. 3, and the energy of the maximum PL intensity of the selective excitation emission shows a change of slope

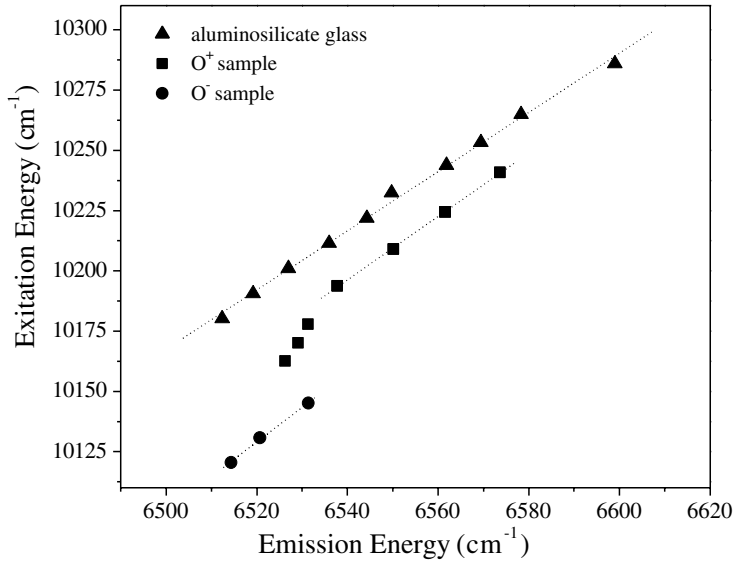

Fig. 5. Maximum intensity of the selective excitation emission as a function of the excitation energy for the aluminosilicate glass, the $\mathrm{O}^{+}$film and the $\mathrm{O}^{-}$film.

near $10180 \mathrm{~cm}^{-1}$ excitation energy (see Fig. 5). For this sample, the site distribution can be divided in two contributions, that suggesting the existence of two kinds of sites. The spectral spreading of this site distribution (around $80 \mathrm{~cm}^{-1}$ ) is reduced compared to aluminosilicate glass and is globally shifted towards longer wavelengths. In opposite to sample $\mathrm{O}^{+}$, the site distribution for sample $\mathrm{O}^{-}$do not present any discontinuity as can be observed in Fig. 4. As was observed for the aluminosilicate glass, the energy of the maximum PL intensity of the selective excitation emission for the $\mathrm{O}^{-}$sample varies linearly with excitation energy (see Fig. 5). However, the site distribution for this sample seems very reduced $\left(25 \mathrm{~cm}^{-1}\right)$ and strongly shifted to longer wavelengths.

Due to the presence of both nitrogen and oxygen in the AlN films, it is reasonable to interpret the two sites distributions observed in $\mathrm{O}^{+}$as being related to Er-O and Er-N environments. Taking into account the covalency of the bondings and its influence on the energy gap between manifolds (nephelauxetic effect [11]) the oxygen site corresponds to the first part of the site distribution $(973.04 \rightarrow 981 \mathrm{~nm})$. It is interesting to note that the spectral range covered by this first site distribution is similar to that of the reference sample. This suggests that it is related to $\mathrm{Er}-\mathrm{O}-\mathrm{Al}$ environment. The "nitrogen" site would then correspond to the second part of the site distribution at longer 
wavelength which agrees the covalent nature of the $\mathrm{Er}-\mathrm{N}$ bonding. It is surprising that this rupture of the site distribution is not observed in the $\mathrm{O}^{-}$ sample. In this sample, it seems that only the nitrogen site is detected with a small site distribution extending from 985.7 to $988.1 \mathrm{~nm}$. However, this film contains around 6.2 at.\% of oxygen and the corresponding site distribution at shorter wavelengths was then expected.

\section{Conclusions}

The aim of this paper was to analyse the environment of erbium ions in AlN films using nonresonant site-selective spectroscopy. Two samples with different oxygen contents have been studied and an influence of oxygen concentration on the site distribution has been observed. It appears that the site distribution is not continuous as in classical aluminosilicate glass but presents a rupture that suggests the existence of two kinds of sites for erbium: "oxygen" site and "nitrogen" site. These observations can easily be interpreted within the framework of the nephelauxetic effect which explains the shift of the site distribution to the longer wavelengths in accordance with an increase of the bonding covalency. It would be further interesting to have access to the crystal field splitting and to the homogeneous broadening of such covalent erbium environment.

\section{Acknowledgements}

The authors would like to thanks the Ambassade de France au Portugal and the Gabinete de Relações Internacionais da Ciência e do Ensino Superior for their joined financial support for missions in France and in Portugal (grant no. 355 B4).

\section{References}

[1] J.M. Zavada, M. Thaik, U. Hommerich, J.D. McKenzie, C.R. Abernathy, S.J. Pearton, R.G. Wilson, J. Alloys Compds. 300 (2000) 207.

[2] M. Fujii, M. Yoshida, Y. Kanzawa, S. Hayashi, K. Yamamoto, Appl. Phys. Lett. 71 (1997) 1198.

[3] P.N. Favennec, H. L'Haridon, D. Moutonnet, Y.L. Guillo, Electron. Lett. 25 (1989) 718.

[4] R.G. Wilson, R.N. Schwartz, C.R. Abernathy, S.J. Pearton, N. Newman, M. Rubin, T. Fu, J.M. Zavada, Appl. Phys. Lett. 65 (1994) 992.

[5] K. Gurumurugan, H. Chen, G.R. Harp, W.M. Jadwisienczak, H.J. Lozykowski, Appl. Phys. Lett. 74 (1999) 3008.

[6] J.C. Oliveira, A. Cavaleiro, M.T. Vieira, Surf. Coatings Technol. 132 (2000) 99.

[7] M. Kuball, Surf. Interface Anal. 31 (10) (2001) 987.

[8] F. Auzel, 1st Franco-Israeli Workshop, Jerusalem, December 1988 (published in SPIE, 1182 (1989) p. 59).

[9] R.M. MacFarlane, R.M. Shelby, J. Luminescence 36 (1987) 179.

[10] L. Bigot, A.M. Jurdyc, B. Jacquier, D. Bayart, L. Gasca, Phys. Rev. B 66 (2002).

[11] R. Reisfeld, C.K. Jorgensen, in: Lasers and Excited States of Rare Earths, Springer-Verlag, 1977. 